\title{
Éducation et nouveau capitalisme en Amérique latine
}

Education and new capitalism in Latin America

Educación y nuevo capitalismo en América latina

Juan Carlos Tedesco

Traducteur : Sophie Blanquer et Rozenn Dedeyan

\section{OpenEdition}

Journals

Édition électronique

URL : http://journals.openedition.org/ries/2387

DOI : $10.4000 /$ ries. 2387

ISSN : 2261-4265

Éditeur

Centre international d'études pédagogiques

\section{Édition imprimée}

Date de publication : 1 octobre 2000

Pagination : 89-100

ISSN : $1254-4590$

\section{Référence électronique}




\section{Éducation \\ et nouveau capitalisme en Amérique latine ${ }^{1}$}

Juan Carlos Tedesco

\section{Résumé}

Dans le contexte de démocratisation des pays d'Amérique latine, l'éducation se trouve au cœur des tensions entre croissance économique et justice sociale. Les réformes éducatives ont privilégié jusqu'à présent l'évolution financière et administrative des systèmes, au détriment des aspects pédagogiques. Le processus d'amélioration qualitative de l'enseignement se trouve freiné par ce choix auquel s'ajoutent les difficultés de concertation entre les différents acteurs.

\section{Education and new capitalism in Latin America}

In the context of increased democracy in Latin American countries education is at the source of conflicts between economic growth and social justice. Until now reforms have favoured the financial and administrative development of the education system to the detriment of teaching. The qualitative improvement of education has been slowed down because of this choice and the problems of communication between the different partners.

\section{Educación y nuevo capitalismo en América latina}

En el contexto de democratización de los países de América latina, la educación es el centro de las tensiones entre crecimiento económico y equidad social. Las reformas educativas han privilegiado hasta ahora una evolución financiera y administrativa. Esta opción económica además de las dificultades de concertación entre los diferentes actores, ha perjudicado el proceso de mejora cualitativa de la enseñanza.

1 Cet article a été traduit par Sophie Blanquer et Rozenn Dedeyan. 
L'Amérique latine a subi un processus de changement politique, économique et social très profond depuis la fin des années soixante-dix. Du point de vue politique, elle a connu un retour à la démocratie qui est actuellement le régime dominant dans la quasi-totalité des pays. Sur le plan économique, après avoir traversé la crise la plus grave depuis la dépression de 1930, la région a entamé un processus de transformation qui a permis le retour à la croissance, le contrôle de l'inflation et un progrès significatif du processus d'intégration régionale et internationale. Du point de vue social, cependant, la croissance économique n'a non seulement pas permis de résoudre les problèmes classiques de pauvreté, mais elle a provoqué une augmentation de l'hétérogénéité structurelle et, en conséquence, des inégalités sociales.

Les nouvelles conditions économiques, politiques et sociales ont amené, par la force des choses, des changements significatifs dans le domaine de l'éducation. De ce fait, l'Amérique latine traverse actuellement une période de réformes en profondeur des systèmes éducatifs, tant au niveau de l'administration du système que de la pédagogie.

La relation qui existe entre éducation et développement social en Amérique latine ainsi que les principaux freins aux processus de changement éducatif en cours dans la plupart des pays feront l'objet de cet article.

\section{Éducation et développement économique et social}

Le modèle classique de développement social en Amérique latine repose sur trois facteurs fondamentaux : l'exploitation - voire la déprédation - des ressources naturelles, la dette extérieure, le déséquilibre financier interne complété par l'inflation. Dans ce contexte, l'une des particularités de l'Amérique latine réside dans le fait que si certains pays ont obtenu une croissance économique, d'autres un niveau minimum de justice sociale, aucun d'entre eux n'est parvenu à atteindre à la fois les deux objectifs du processus de développement : croissance et justice. Les études réalisées sur cette question montrent qu'entre 1970 et 1984, les pays qui jouissaient de taux de croissance du PIB supérieurs à $2 \%$ maintenaient des niveaux de concentration de la richesse très élevés, tandis que les pays où le degré de justice sociale était relativement satisfaisant ne parvenaient pas à croître économiquement ${ }^{2}$.

Dans ce modèle de développement, l'éducation a été caractérisée par un très fort décalage par rapport à la demande du développement socioéconomique. Les résultats de l'éducation n'ont presque pas eu de rapport avec les activités économiques, à cause de l'utilisation limitée du progrès technique comme facteur de production, ni avec l'intégration politique, parce que l'intégration des

2 F. Fajnzylber, Industrialización en América Latina : de la "Caja Negra " al "Casillero Vacío ", Santiago du Chili, Cepal, 1989. 
citoyens a été constamment freinée par de longues périodes d'autoritarisme et d'exclusion. Ce processus a conduit à la consolidation d'une offre éducative très large, mais associée à des insuffisances criantes du point de vue de la qualité des résultats et des mécanismes de gestion institutionnelle.

Les indicateurs les plus manifestes de ce modèle de développement éducatif sont les taux élevés de scolarisation, accompagnés par des taux tout aussi élevés de redoublement et de faibles niveaux de réussite dans les apprentissages, qui affectent essentiellement les élèves issus des secteurs les plus économiquement défavorisés. Ainsi, par exemple, le taux de scolarisation primaire (6-11 ans) est passé de 57,7 en 1960 à 87,5 en 1990, celui de scolarisation secondaire (12-17 ans) de 36,3 à 66,2 et le taux de fréquentation de l'enseignement supérieur (18-23 ans) de 5,7 à 27. En même temps, le taux moyen de redoublement de la première année d'école primaire pour l'Amérique latine et les Caraïbes était de $42 \%$ en 1990. Certaines études effectuées par l'UNESCO montrent que le redoublement touche plus de 20 millions d'élèves et coûte environ 4,2 milliards de dollars par an ${ }^{3}$.

Dans le même sens, les résultats des études effectuées pour mesurer la maîtrise des apprentissages montrent non seulement des résultats faibles pour l'ensemble des pays, mais aussi des différences importantes entre les apprentissages maîtrisés par les élèves qui fréquentent des écoles privées d'élite et les élèves qui fréquentent des écoles publiques dans des zones défavorisées. L'étude pilote TIMSS ${ }^{4}$, destinée à évaluer les résultats des élèves âgés de treize ans dans cinq pays d'Amérique latine (Argentine, Colombie, Costa Rica, République dominicaine et Venezuela), a montré que les élèves des écoles privées d'élite obtenaient des résultats de 50 à $75 \%$ supérieurs à ceux des élèves des écoles publiques économiquement défavorisées ${ }^{5}$.

Ce modèle traditionnel de développement économique et éducatif a donné de nets signes d'épuisement à la fin des années quatre-vingt. Il existe de nombreuses analyses sur l'origine et l'impact de la crise économique durant cette période, où les débats furent centrés sur la question de la dette extérieure. Certains, à court terme, tendaient à considérer cette situation de crise comme un problème cyclique et non comme l'épuisement d'un modèle de développement. Le financement de l'éducation ne fut pas perçu comme un facteur qui devait être préservé à cause de son importance stratégique. L'investissement public dans l'éducation fut réduit, entre 1980 et 1985, de 33,5 milliards à 27,9 milliards de dollars. Les études sur cette question montrent que la princi-

3 E. Schiefelbein, Juan Carlos Tedesco, Una Nueva Oportunidad : Educación y Desarrollo en América Latina, Buenos Aires, Santillana, 1994.

4 Third International Mathematics and Science Study.

5 E. Schiefelbein, "Reforma Educativa en América Latina y el Caribe : Una Agenda para la Acción ", Proyecto Principal de Educación en América Latina y el Caribe, $n^{\circ}$ 37, 1995. 
pale réduction porta sur le salaire des enseignants, sans épargner pour autant les sommes destinées à l'équipement, à l'entretien des bâtiments scolaires et à la formation du personnel.

La perception de la crise comme un problème conjoncturel et la tendance à la réduction de l'investissement public dans l'éducation commencèrent à être dépassées au début des années quatre-vingt-dix ${ }^{6}$. Ce changement se produisit dans un contexte de profonde transformation de la production et de réforme de l'État, où les deux thèmes les plus discutés étaient la justice sociale et le rôle de l'État. Le débat était alimenté par la confrontation de deux visions opposées.

Du point de vue de ceux qui considèrent la justice sociale comme un objectif essentiel du développement social, l'accent a été mis sur une conception globale selon laquelle la justice sociale ne doit pas être conçue comme un facteur extérieur au processus de croissance économique, mais comme une variable dont les effets sur la production et les institutions sont fondamentaux pour assurer une croissance économique soutenue.

Selon cette approche théorique, élaborée et diffusée dans la région à travers les analyses effectuées par la CEPAL ${ }^{7}$, la variable qui permettrait d'articuler et de rendre compatibles les objectifs de croissance et de justice est le progrès technique. Une croissance économique sans progrès technique impliquerait le maintien de la compétitivité par la diminution des salaires et l'exploitation abusive des ressources naturelles. Les analyses sur les perspectives économiques mondiales indiquent, cependant, que ces facteurs ne peuvent assurer un développement économique soutenu pour deux raisons essentielles : d'une part, le faible coût de la main-d'œuvre joue un rôle de moins en moins important dans l'explication de la productivité économique et, d'autre part, les exigences de protection de l'environnement sont devenues un facteur de plus en plus important dans les processus d'intégration au commerce international. Mais, à l'inverse, justice sociale et protection de l'environnement sans progrès technique seraient également des objectifs impossibles à atteindre, parce que cela impliquerait l'arrêt de la croissance économique et provoquerait un isolement incompatible avec le niveau de développement et les attentes sociales qui existent dans la majorité des pays de la région.

Selon cette optique, l'intégration du progrès technique à la production implique de mener à bien des actions systématiques et délibérées dans divers domaines dont, précisément, l'éducation. L'idée d'une intervention délibérée et systématique est en particulier liée au rôle de l'État qui, de ce point de vue, devrait jouer un rôle actif.

6 Les données disponibles indiquent qu'entre 1985 et 1995 les dépenses publiques consacrées à l'éducation ont doublé, passant de 27,9 à 72,8 millions de dollars.

7 CEPAL, Transformación productiva con equidad. La tarea prioritaria del desarrollo de América Latina y el Caribe en los años noventa, Santiago du Chili, CEPAL, 1990. 
Pour que l'éducation contribue effectivement au progrès technique, dans le contexte des évolutions scientifiques et technologiques actuelles, il faut qu'elle modifie considérablement ses modèles de fonctionnement. Il ne s'agit pas, en conséquence, d'élargir l'offre éducative traditionnelle. L'application de nouveaux schémas de développement exige que soient définies des stratégies de changement en matière d'éducation, orientées non seulement vers une expansion quantitative mais aussi vers la qualité des résultats de la formation ${ }^{8}$.

Cette conception de l'articulation entre éducation et développement social s'est vu opposer une approche différente, selon laquelle la justice sociale et la compétitivité économique ne seraient pas le résultat de politiques actives de l'État mais plutôt de la dérégulation des activités économiques. Selon ce point de vue, le marché serait capable, par lui-même, d'établir la meilleure distribution des ressources. Cette confiance dans le marché a été également préconisée pour l'éducation, où la stratégie la plus importante devrait être la privatisation, le financement de la demande et l'introduction de mécanismes de concurrence entre les écoles.

Les transformations de la production ont été liées à la dérégulation, d'une part, et à la réforme en profondeur de l'État, d'autre part. Le retour à la démocratie en Amérique latine s'est produit quand l'État n'a plus été en mesure de satisfaire les demandes sociales.

À partir de cette représentation schématique de la relation entre éducation et développement, il est possible d'effectuer l'analyse des processus de réforme éducative actuellement en vigueur en Amérique latine, selon trois axes de discussion qui peuvent résumer les principaux débats éducatifs actuels : l'éducation et la justice sociale; la réforme éducative; la concertation en matière de politiques éducatives.

\section{La problématique de la transformation éducative en Amérique latine}

\section{Éducation et justice sociale}

L'expérience des dernières années a montré que la relation entre éducation et justice sociale à travers le progrès technique n'est pas aussi directe que ne l'avaient prévu les analyses de la CEPAL. Le progrès technique requiert, bien évidemment, des ressources humaines qualifiées, mais il détruit aussi plus de postes de travail qu'il n'est capable d'en créer. Les taux de chômage ont augmenté de façon très significative et la répartition de la richesse est aujourd'hui beaucoup moins équitable que par le passé.

8 ECLAC-UNESCO, Education and Knowledge : Basic Pillars of Changing Production Patterns with Social Equity. Santiago du Chili, CEPAL, 1992. 
D’après les études réalisées sur la distribution de la richesse, la croissance économique de la dernière décennie a été associée à une augmentation de son caractère inéquitable. L'Amérique latine est, selon ces études, la région qui présente les plus hauts niveaux de concentration du revenu dans le monde (voir tableau 1).

Tableau 1

Répartition du revenu mondial en 1990

\begin{tabular}{|l|c|c|c|c|c|c|}
\hline Région & $\begin{array}{c}\text { Afrique } \\
\text { du Nord } \\
\text { et Moyen- } \\
\text { Orientile* }\end{array}$ & $\begin{array}{c}\text { Amérique } \\
\text { latine }\end{array}$ & $\begin{array}{c}\text { Asie } \\
\text { du Sud }\end{array}$ & $\begin{array}{c}\text { Asie } \\
\text { du } \\
\text { Sud-Est }\end{array}$ & $\begin{array}{c}\text { Europe } \\
\text { de l'Ouest }\end{array}$ & $\begin{array}{c}\text { OCDE } \\
\text { et pays } \\
\text { à hauts } \\
\text { revenus }\end{array}$ \\
\hline 1 & 6,90 & 4,52 & 8,76 & 6,84 & 8,83 & 6,26 \\
\hline 2 & 10,91 & 8,70 & 12,91 & 11,30 & 13,36 & 12,15 \\
\hline 3 et 4 & 36,84 & 33,84 & 38,42 & 37,53 & 40,01 & 41,80 \\
\hline 5 & 45,35 & 52,94 & 39,91 & 44,33 & 37,80 & 39,79 \\
\hline
\end{tabular}

Source : Deininger et Squire, « Measuring Income Inequality. A New Data-base ", World Economic Review.

* Les quintiles correspondent à des mesures partageant une distribution en cinq parties égales.

Dans ce contexte, il y a lieu de rouvrir le débat sur le rôle de l'éducation. Si l'on admet que l'éducation est un facteur important de justice sociale, la question inverse a également commencé à se poser : quel niveau de justice sociale faut-il pour permettre une éducation de bonne qualité ? Les inégalités croissantes provoquent un phénomène de détérioration des conditions d' " éducabilité » grâce à laquelle les élèves ont accès à l'éducation. Les données sur la maitrise des apprentissages des élèves indiquent que l'offre éducative ne peut compenser les déficits de socialisation de base des élèves issus de familles pauvres (voir tableau 2).

Tableau 2

Moyenne des résultats scolaires en fonction du niveau socioéconomique (Quatrième année de l'enseignement de base)

\begin{tabular}{|l|c|c|c|}
\hline Résultats & $\begin{array}{c}\text { Niveau socio- } \\
\text { économique faible }\end{array}$ & $\begin{array}{c}\text { Niveau socio- } \\
\text { économique moyen }\end{array}$ & $\begin{array}{c}\text { Niveau socio- } \\
\text { économique élevé }\end{array}$ \\
\hline Langue & 47,9 & 58,4 & 71,9 \\
\hline Mathématiques & 43,8 & 49,8 & 59 \\
\hline Total & 46 & 54,1 & 65,5 \\
\hline
\end{tabular}

Source: OREALC-UNESCO.

Dans ce contexte, les réformes éducatives en Amérique latine doivent faire face non seulement aux demandes provenant des nouvelles exigences de la fonction de production mais encore aux demandes de cohésion sociale posées par les vastes secteurs de population exclus de l'accès à l'emploi et à la richesse. 
Le risque le plus évident de cette dynamique est l'augmentation de la fracture et de la polarisation éducative qui s'observe dans tous les pays de la région à travers, notamment, les pratiques politiques, certains discours et stratégies d'action - tendance selon laquelle l'État ne devrait s'occuper que des pauvres et le secteur privé des besoins plus sophistiqués et complexes des couches moyennes et supérieures de la population.

L'expérience de cette décennie permet d'affirmer que l'objectif d'une plus grande justice sociale par le biais de l'éducation ne dépend pas seulement de changements dans l'offre pédagogique. L'équité est un phénomène systémique et, par conséquent, sans modifications substantielles des modèles de distribution des revenus, il sera impossible d'avancer vers les objectifs éducatifs qui permettraient à la population d'avoir accès à des niveaux d'éducation suffisants pour son intégration productive à la société.

\section{La réforme éducative}

Parmi toutes les étapes potentielles du processus de transformation éducative, les pays de la région ont choisi de commencer par un changement institutionnel. En termes plus concrets, il s'agit de décentralisation et, dans certains cas, d'une plus grande autonomie accordée aux écoles.

Décentralisation et autonomie des établissements scolaires ont été historiquement revendiquées par certains éducateurs et mouvements pédagogiques qui souhaitaient une plus grande liberté des acteurs du processus éducatif afin d'innover, de faire des propositions de curricula et de s'adapter à la diversité sociale et culturelle des élèves. Mais cette tradition pédagogique a perdu de son importance au cours des années quatre-vingt, la décentralisation a été mise en ouvre d'un point de vue administratif et budgétaire, en se focalisant sur la réduction des dépenses publiques, l'utilisation plus efficace des ressources disponibles et l'affaiblissement du pouvoir de négociation des syndicats d'enseignants à cause de la fragmentation des domaines de négociation.

Le débat sur les processus de décentralisation est intense et bien connu et il ne s'agit pas ici de traiter de ce problème. Il nous paraît seulement intéressant de noter un fait évident mais qui, paradoxalement, n'est pas toujours pris en compte dans ces discussions : les pays décentralisés n'ont pas d'expérience de décentralisation parce qu'ils ont toujours été décentralisés, et les pays centralisés mais riches font leur expérience de la décentralisation dans le cadre d'une grande disponibilité de ressources et d'une grande homogénéité sociale. Les pays d'Asie du Sud-Est, constamment cités comme modèle à suivre pour les pays en voie de développement, ont réussi leur expérience éducative sans réforme institutionnelle de ce type. 
En définitive, la discussion sur les processus de décentralisation dans des contextes de pauvreté oblige à se demander si la concurrence entre institutions et agents est compatible avec l'équité et si la décentralisation est une garantie de dynamisme et de participation démocratique.

Il est intéressant de souligner qu'en Amérique latine on est parti de l'hypothèse qu'il fallait commencer par changer les institutions avant tout autre domaine : contenu des curricula, méthodes pédagogiques, formation des enseignants, équipement des écoles, conditions de travail des enseignants, etc.

Malgré l'immense diversité des situations existant dans la région, ce schéma s'est généralisé presque partout tant entre pays qu'à l'intérieur de chacun d'eux. On peut donc parler d'un degré d'uniformité excessif dans les processus de réforme éducative, qui a entraîné un certain nombre de conséquences, dont on citera deux exemples.

En premier lieu, l'appauvrissement de la notion de transformation éducative, qui tend à se réduire aux aspects financiers ou administratifs. Ce phénomène a été constaté dans plusieurs études effectuées auprès de cadres moyens et supérieurs des administrations éducatives locales qui expriment de grandes difficultés à conceptualiser ce qu'ils font, à projeter dans l'avenir, à anticiper certaines situations et à capitaliser leur expérience. Ce sentiment d'une «absence de sens» se retrouve parfois également chez les enseignants et dans certains secteurs de l'opinion publique. Dans le cas des enseignants, en particulier, l'absence d'information ou de compréhension par rapport au sens du processus global de changement donne le sentiment que la transformation est fondamentalement liée à l'idée de perte et à des sentiments d'insécurité et d'incertitude pour l'avenir.

En second lieu, la priorité donnée aux aspects institutionnels semble avoir fait oublier les aspects pédagogiques et les principaux acteurs de ce processus, c'est-à-dire les enseignants. Dans ce sens, il faut reconnaître qu'une bonne partie des débats qui ont eu lieu au cours des dernières décennies sur les processus de transformation éducative ont ignoré, sous-estimé ou tout simplement envisagé de manière purement formelle le rôle des enseignants. Ce manque de considération pour le rôle de l'enseignant, dont le bas niveau de salaire est un des symptômes, provoque des situations de résistance aux processus de transformation éducative qui empêchent d'avancer au rythme voulu par les nouvelles demandes sociales. Cependant, les résistances manifestées par les enseignants ne devraient pas être analysées exclusivement comme étant le résultat de leurs mauvaises conditions de travail. Il existe des variables techniques et culturelles dont il ne faut pas sous-estimer l'importance. Diverses études ont montré que les enseignants ou, du moins, une grande partie d'entre eux, perçoivent le processus de globalisation comme une menace dans ses aspects liés à la crise de l'État- 
nation et à la culture des nouvelles technologies de l'information. De la même manière, les enseignants ne maîtrisent pas toujours certaines compétences qu'on leur demande de diffuser : créativité, capacité à travailler en équipe, grande résistance à l'incertitude, capacité à élaborer des projets, etc. Par ailleurs, l'intégration à l'école de catégories sociales et culturelles très diverses exige le maniement d'une batterie de méthodes très vaste, à même de s'adapter aux situations de diversité culturelle et sociale, que la pédagogie et la formation des enseignants dans les pays en développement n'ont pas encore réussi à élaborer.

En résumé, tout indique que les réformes éducatives devraient, à l'avenir, accorder davantage d'attention aux aspects proprement pédagogiques et que la pédagogie du futur devra avoir une forte dimension culturelle.

\section{La concertation des politiques éducatives}

La nécessité d'élaborer les stratégies d'action éducative avec la participation de tous les acteurs sociaux est déjà un lieu commun dans le discours sur les politiques éducatives. La continuité dans l'application des stratégies de transformation a été reconnue comme l'une des conditions de leur réussite et, pour qu'il y ait continuité, tout au moins dans des contextes démocratiques, il est nécessaire qu'existe un niveau minimum d'accord et d'engagement de tous les acteurs sur cette mise en œuvre. À cet égard, au début des années quatre-vingt-dix, un certain optimisme prévalait quant à l'existence de conditions favorables à la définition de stratégies éducatives et consensuelles.

Le facteur-clé de cet optimisme était la constatation selon laquelle, dans les nouveaux scénarios sociaux, la connaissance et l'information constituaient les variables essentielles tant du point de vue de la compétitivité économique que du sens civique et de la justice sociale. Néanmoins, il s'avéra rapidement que pour aller au-delà de la simple reconnaissance rhétorique de la nécessité de concertation sur le plan des politiques éducatives, il fallait surmonter de très importantes difficultés. Si certaines d'entre elles, propres aux pays en voie de développement, tiennent à cette absence de culture de concertation, elles semblent puiser leurs sources dans :

- la position centrale qu'occupe la connaissance dans la structure sociale, puisque, dans la mesure où l'information et la connaissance constituent de plus en plus les variables-clés de la répartition du pouvoir, le contrôle tant de leur production que de leur diffusion devient l'enjeu des conflits sociaux les plus importants ; 
- la forte inégalité sociale en Amérique latine qui provoque un manque significatif de confiance parmi les différentes catégories sociales vis-à-vis des règles de concertation sociale et politique ${ }^{9}$.

Dans ce contexte, la concertation sur les politiques éducatives ne peut être un processus exempt de conflits. Au contraire, l'expérience récente indique que la concertation est un processus difficile et complexe, ce qui peut s'expliquer par un certain nombre de raisons.

En premier lieu, la difficulté d'avoir une représentation adéquate des secteurs socialement défavorisés dans les négociations sur l'éducation. Les expériences récentes de concertation éducative ont montré que la phase des processus de concertation repose sur un processus de participation de plus en plus corporatiste. Dans la phase initiale, qui est généralement une phase de consultation, de discussion, de diagnostic, la participation est habituellement très large. Des événements comme le congrès pédagogique d'Argentine, le jour " J » de l'éducation au Brésil, la consultation populaire sur l'éducation en Équateur, la consultation en République dominicaine sont autant d'exemples de processus de mobilisation de l'opinion publique qui ont généré de grandes attentes. Quand la discussion arrive à la phase de définition d'engagements et de modalités d'action, la diversité des participants tend à diminuer et la participation prend un caractère plus corporatiste. La représentation des secteurs les plus défavorisés tend à disparaître ou à être récupérée par telle ou telle organisation politique, ou par l'Église, avec toutes les conséquences que cela peut impliquer en termes d'utilisation de cette représentation pour servir d'autres intérêts.

En second lieu, les expériences montrent qu'il existe une différence importante entre les accords auxquels on parvient sur des objectifs généraux et les accords concernant la mise en œuvre. Les témoignages sur ce point sont éloquents : arriver à un consensus au cours de la phase de définition des objectifs est relativement facile par rapport aux énormes difficultés que l'on rencontre pour arriver à un consensus dans la phase d'exécution. Ce phénomène est lié au fait que les possibilités de garantir les accords obtenus au cours de la phase précé-

9 Diverses études montrent que le phénomène du manque de confiance a pris des proportions très significatives en Amérique latine. Entre novembre et décembre 1997, une enquête a été menée dans dix-sept pays par la Corporación Latinbarómetro: seulement $23 \%$ des personnes interrogées pensent que leur pays progresse. La confiance dans les institutions détenant le plus de pouvoir (gouvernement, grandes entreprises, armée, banques, partis politiques) est très faible. Dans les pays connaissant un début de démocratisation, un très grand nombre de personnes sont partisanes d'un régime autoritaire et, mis à part en Uruguay et au Costa Rica, $65 \%$ des personnes interrogées se sont montrées peu ou pas du tout satisfaites de la démocratie. Le cas du Chili est aussi éclairant. Une enquête préparée pour le rapport du PNUD indiquait des niveaux élevés d'insatisfaction. Malgré la croissance économique soutenue, il existe un sentiment de malaise croissant qui se manifeste à travers la peur de l'exclusion, de l'autre et de l'" absence de sens ". "Les gens n'ont pas confiance dans l'avenir. "Dans le cas de l'Argentine, une enquête récente effectuée auprès de jeunes et d'adultes dans la région de Buenos Aires a révélé une grande méfiance vis-à-vis des institutions publiques. 
dant l'application sont bien moindres dans les pays en voie de développement. La faiblesse des moyens oblige à définir des priorités laissant une faible marge de manœuvre pour satisfaire les demandes des différents secteurs. Comme cette pénurie des ressources perdure sur le long terme, l'urgence des demandes est très significative. Dans ce contexte, il est difficile de faire accepter des processus de changement qui impliquent de remettre à plus tard la satisfaction de certaines demandes. Personne n'est disposé à attendre plus longtemps et, bien évidemment, dans ce conflit d'urgences ceux qui sont à même de faire valoir leurs intérêts sont les secteurs les mieux organisés. L'un des indicateurs les plus significatifs de cette capacité inégale de négociation est la répartition des ressources financières par niveau d'instruction. L'enseignement supérieur, qui compte sur la capacité de mobilisation des étudiants des universités, représente un pourcentage de plus en plus important dans les dépenses consacrées à l'éducation.

En troisième lieu, il faut mentionner la multiplication des acteurs qui prennent part aux processus de concertation éducative. Contrairement à la situation traditionnelle, où le débat éducatif se concentrait sur l'État, les enseignants et l'Église, on assiste à l'apparition de nouveaux acteurs sociaux intéressés à participer et à discuter des stratégies éducatives. Ces nouveaux acteurs viennent du monde du travail (les chefs d'entreprise participent de plus en plus aux débats sur l'éducation); des médias (le journalisme s'intéresse davantage à l'école et les moyens de communication de masse font l'objet d'un débat concernant leur rôle comme agents de l'éducation); enfin, il faut signaler la présence - particulièrement importante dans les pays en voie de développement - d'acteurs nouveaux, liés au processus de globalisation : les organismes internationaux de financement, de coopération et de recherche en matière d'éducation. Les organismes de financement ont assumé une responsabilité croissante dans l'orientation des stratégies de changement et les spécialistes de l'éducation sont de plus en plus présents dans les organismes de prise de décisions. De fait, un nombre important de ministres et de hauts fonctionnaires de l'éducation sont, ou ont été, des chercheurs reconnus dans le monde universitaire et éducatif.

En résumé, tout semble indiquer que l'on a largement dépassé la vision «angélique » des processus de concertation et de participation et que l'on a pris conscience de ce que ces processus donnent lieu à des alliances et à des conflits où, au-delà des accords, des intérêts différents continuent de coexister.

\section{Les défis pour l'avenir}

Cette analyse sommaire de la situation et des perspectives d'éducation en Amérique latine permet d'affirmer que les défis auxquels il faudra faire face dans un proche avenir se rapportent à trois objectifs principaux : la justice sociale, la compétitivité économique et le civisme politique. L'éducation est probablement la seule politique publique qui produise des effets simultanés sur ces trois objectifs. 
Du point de vue de la justice sociale, les lignes politiques sur lesquelles il serait nécessaire de mettre l'accent concernent l'élargissement des programmes compensatoires, la généralisation de l'éducation préscolaire et des primes à l'innovation, l'amélioration de la qualité de l'offre éducative pour les groupes les plus défavorisés et le renforcement de la capacité des secteurs en difficulté à effectuer des demandes éducatives plus qualifiées.

Du point de vue de la compétitivité, chacun s'accorde à reconnaître la nécessité urgente d'améliorer les niveaux de qualité des résultats éducatifs, en tenant compte des exigences de la mise en œuvre des processus de transformation de la production. Des modifications dans les contenus et les méthodes pédagogiques destinés à généraliser les compétences de base dans un but de production sont déjà en cours dans divers pays de la région. De la même façon, le défi de la compétitivité implique d'introduire une meilleure articulation entre l'éducation et le monde du travail, en particulier par l'utilisation des nouvelles technologies de l'information.

Enfin, quant à l'éducation à la citoyenneté, le défi pour les systèmes éducatifs en Amérique latine consistera à introduire dans les programmes des expériences de comportement démocratique, de respect de la diversité culturelle et une forte promotion de valeurs centrées sur la solidarité sociale. Le manque de justice sociale dans la région est à la base de la fragilité de la démocratie. S'attaquer à ce problème exigerait, du point de vue des valeurs culturelles, un fort engagement de solidarité que le système éducatif doit contribuer à développer, particulièrement au sein des élites qui concentrent la richesse. 